\title{
CONTROLADOR EM MODO DUAL ADAPTATIVO ROBUSTO PARA PLANTAS COM GRAU RELATIVO ARBITRÁRIO
}

\author{
Caio D. Cunha* \\ dornelesdufrnet.br
}

\author{
Aldayr D. Araújo* \\ aldayredca.ufrn.br
}

\author{
Francisco C. Mota ${ }^{\dagger}$ \\ mota@dca.ufrn.br \\ * Departamento de Engenharia Elétrica -UFRN \\ CEP:59.072-970 Natal-RN, fone 843215 3731-fax 8432153767 \\ ${ }^{\dagger}$ Departamento de Engenharia da Computação e Automação -UFRN CEP:59.072-970 Natal-RN, fone 843215 3771-fax 84
32153738
}

\section{RESUMO}

Neste artigo é apresentado o Controlador em Modo Dual Adaptativo Robusto (DMARC). O DMARC é um sistema de controle que combina as estratégias de Controle Adaptativo por Modelo de Referência (MRAC) com leis integrais de adaptação e o Controle Adaptativo por Modelo de Referência e Estrutura Variável (VS-MRAC) com leis chaveadas de acordo com a teoria de sistemas com estrutura variável. A idéia básica é incorporar as vantagens de desempenho transitório de um VS-MRAC, com as propriedades de regime permanente de um MRAC convencional. Desta forma, obtém-se um sistema de controle robusto a incertezas paramétricas e perturbações externas, com desempenho rápido e pouco oscilatório durante o transitório e um sinal de controle suave em regime permanente.

PALAVRAS-CHAVE: Controle em Modo Dual, Controle Adaptativo, Sistemas com Estrutura Variável.

\section{ABSTRACT}

In this paper the Dual-Mode Adaptive Robust Controller (DMARC) is presented. The DMARC is a control strategy which interpolates the Model Reference Adaptive Control

Artigo submetido em 16/12/2004 (Id:608)

Revisado em 19/07/2006, em 10/04/2008 e em 27/11/2008

Aceito sob recomendação do Editor Associado Prof. Liu Hsu
(MRAC) and the Variable Structure Model Reference Adaptive Control ( VS-MRAC). The main idea is to develop a robust controller to parametric uncertainties and external disturbances with good transient characteristics (fast response and small oscillations) as in a VS-MRAC and good steadystate characteristics (smooth control signal) as in a conventional MRAC.

KEYWORDS: Dual Mode Control, Adaptive Control, Variable Structure Systems.

\section{INTRODUÇÃO}

O Controlador em Modo Dual Adaptativo Robusto teve sua motivação a partir do artigo de Hsu e Costa (1989), onde os autores propuseram uma lei de controle que, dependendo da escolha de um parâmetro, podia se comportar como o Controlador MRAC ou como o Controlador VS-MRAC e suas versões intermediárias.

No MRAC convencional, que usa leis integrais de adaptação, a saída da planta segue um modelo de referência especificado (Ioannou e Sun (1996), Åström e Wittenmark (1995), Sastry e Bodson (1989) e Narendra e Annaswamy (1989)). Mesmo com as modificações para aumentar a robustez do algoritmo convencional (fator $\sigma$, normalização, etc.), em geral o transitório é lento e oscilatório. 
No VS-MRAC, utiliza-se a estrutura do controle por modelo de referência do MRAC. As leis integrais de adaptação são substituídas por leis chaveadas, resultando em um sinal de controle chaveado, como nos sistemas a estrutura variável (ver seção 4) (Hsu e Costa (1989), Hsu, Araújo e Costa (1994), Hsu, Lizarralde e Araújo (1997), Cunha e outros (2003)). Apesar do bom desempenho transitório, em geral tem-se a presença do fenômeno de "chattering".

A partir da teoria de controle binário desenvolvida por Emelyanov (1987), Hsu e Costa propuseram um Controlador Binário Adaptativo por Modelo de Referência (B-MRAC) (Hsu e Costa (1990) e (Hsu e Costa (1994)). De acordo com o princípio binário, existem variáveis que são denominadas coordenadas (sofrem transformações) e variáveis que são denominadas operadores (definem as transformações aplicadas às coordenadas), e são todas tratadas como variáveis de estado do sistema (Andrievskii, Stotskii e Fradkov (1988) e Hsu e Costa (1994)). No B-MRAC é proposta uma combinação de um sistema com estrutura variável e um algoritmo de adaptação paramétrica. Utiliza-se uma lei gradiente de adaptação de alto ganho com projeção, a qual com um parâmetro (operador, segundo o princípio binário) suficientemente elevado faz o controlador tender ao VS-MRAC.

O DMARC faz uma ligação entre o VS-MRAC e o MRAC convencional. O objetivo é conseguir um sistema robusto, com desempenho rápido e pouco oscilatório (características do VS-MRAC), e sinal de controle suave em regime permanente (características do MRAC). A transição entre as duas estratégias de controle é feita, em tempo real, através de um parâmetro (operador) que varia em função do erro entre a saída da planta e a saída do modelo de referência.

A estrutura do controlador, aqui utilizada, baseia-se no VSMRAC generalizado proposto por Araújo e Hsu, (1990) e do B-MRAC proposto por Hsu e Costa (1994).

\section{FORMULAÇÃO DO PROBLEMA}

Considere uma planta linear, com parâmetros incertos, monovariável e invariante no tempo, com entrada $u$ e saída $y$, com função de transferência

$$
W(s)=k_{p} \frac{N_{p}(s)}{D_{p}(s)} .
$$

Considere, também, o modelo de referência tendo a entrada $r$ e a saída $y_{m}$, caracterizado pela função de transferência

$$
M(s)=k_{m} \frac{N_{m}(s)}{D_{m}(s)} .
$$

Tem-se como objetivo determinar $u$ tal que o erro de saída

$$
e_{0}=y-y_{m}
$$

tenda a zero assintoticamente para condições iniciais arbitrárias e sinais de referência $r(t)$ uniformemente limitados e contínuos por partes.

As seguintes hipóteses convencionais são feitas (Sastry e Bodson (1989), p. 103-104):

a. A planta é controlável e observável com grau $\left[D_{p}(s)\right]=n$ e grau $\left[N_{p}(s)\right]=m, n$ e $m$ conhecidos;

b. $\operatorname{sinal}\left(k_{p}\right)=\operatorname{sinal}\left(k_{m}\right)$, positivos por simplicidade;

c. $N_{p}(s)$ é Hurwitz ( $W(s)$ é de fase mínima);

d. O modelo de referência tem o mesmo grau relativo da planta $\left(n^{*}=n-m\right)$;

e. Somente a entrada e a saída da planta são usadas para gerar $u$.

São usados os seguintes filtros de entrada e saída da planta

$$
\left\{\begin{array}{c}
\dot{v}_{1}=\Lambda v_{1}+g u \\
\dot{v}_{2}=\Lambda v_{2}+g y
\end{array}\right.
$$

onde $v_{1}$ e $v_{2}$ pertencem ao $\Re^{n-1}$, e $\Lambda$ é escolhido tal que $N_{m}(s)$ é um fator de det (sI $\left.-\Lambda\right)$. Define-se o vetor regressor como $\omega^{T}=\left[\begin{array}{llll}v_{1}^{T} & y & v_{2}^{T} & r\end{array}\right]$. O controle é, então, definido como

$$
u=\theta(t)^{T} \omega(t)
$$

onde $\theta(t)^{T}=\left[\begin{array}{llll}\theta_{v 1}^{T}(t) & \theta_{n}(t) & \theta_{v 2}^{T}(t) & \theta_{2 n}(t)\end{array}\right]$ é o vetor de parâmetros adaptativos (Ioannou e Sun (1996), p. 384).

Sabe-se que, sob as hipóteses acima, existe um único vetor constante $\theta^{*}$ tal que a função de transferência da planta em malha fechada (com $u=\theta^{*^{T}} \omega$ ), de $r$ para $y$, é $M(s)$ (condição de "Matching"). Obviamente $\theta^{*}$ somente pode ser conhecido se a planta for conhecida. Quando isto não é o caso, $\theta(t)$ é adaptado até que $e_{0}(t) \rightarrow 0$ quando $t \rightarrow \infty$ e, eventualmente sob alguma condição de riqueza de sinal, $\theta(t) \rightarrow \theta^{*}$ (Ioannou e Sun (1996). p. 177).

Seja $\left[A, b, h^{T}\right]$ uma realização mínima da planta e $x \in$ $\Re^{n}$ o respectivo vetor de estado. Então, a planta com os filtros podem ser representados como 


$$
\begin{gathered}
\dot{x}_{c}=A_{0} x_{c}+b_{0} u \\
y=h_{c}^{T} x_{c}
\end{gathered}
$$

onde $x_{c}^{T}=\left[x^{T}, v_{1}^{T}, v_{2}^{T}\right], x_{c} \in \Re^{3 n-2}$,

$$
\begin{aligned}
A_{0}= & {\left[\begin{array}{ccc}
A & 0 & 0 \\
0 & \Lambda & 0 \\
g h^{T} & 0 & \Lambda
\end{array}\right], \quad b_{0}=\left[\begin{array}{l}
b \\
g \\
0
\end{array}\right] } \\
& \text { e } h_{c}^{T}=\left[\begin{array}{lll}
h^{T} & 0 & 0
\end{array}\right] .
\end{aligned}
$$

Nota-se que $\omega=\Omega x_{c}+b_{r} r$ com

$$
\Omega=\left[\begin{array}{c}
\Omega_{c} \\
0
\end{array}\right], \quad \Omega_{c}=\left[\begin{array}{ccc}
0 & I & 0 \\
h^{T} & 0 & 0 \\
0 & 0 & I
\end{array}\right] \quad \text { e } \quad b_{r}=\left[\begin{array}{l}
0 \\
0 \\
0 \\
1
\end{array}\right]
$$

Tem-se, então,

$$
\begin{gathered}
\dot{x}_{c}=A_{c} x_{c}+\frac{b_{c}}{\theta_{2 n}^{*}}\left(u-\theta^{*^{T}} \omega\right)+b_{c} r \\
y \stackrel{h_{c}^{T} x_{c}}{ }
\end{gathered}
$$

onde $A_{c}=A_{0}+b_{0} \theta_{r}^{*^{T}} \Omega_{c}, \theta_{r}^{*^{T}}=\left[\theta_{1}^{*} \ldots \theta_{2 n-1}^{*}\right], b_{c}=$ $\theta_{2 n}^{*} b_{0}, \theta_{2 n}^{*}=k_{m} / k_{p}>0$.

$\left[A_{c}, b_{c}, h_{c}^{T}\right]$ é uma realização não mínima e estável de $M(s)$ (Sastry, (1984)), ou seja, o modelo de referência pode ser representado como

$$
\begin{gathered}
\dot{x}_{c m}=A_{c} x_{c m}+b_{c} r, \quad x_{c m} \in \Re^{3 n-2} . \\
y_{m}=h_{c}^{T} x_{c m} .
\end{gathered}
$$

Definindo o vetor de erro por $e=x_{c}-x_{c m}$, tem-se a seguinte equação de erro

$$
\begin{gathered}
\dot{e}=A_{c} e+\frac{b_{c}}{\theta_{2 n}^{*}}\left(u-\theta^{*^{T}} \omega\right) \\
e_{0}=h_{c}^{T} e
\end{gathered}
$$

$$
e_{0}=\frac{1}{\theta_{2 n}^{*}} M\left(u-\theta^{*^{T}} \omega\right)
$$

Observe que nenhuma restrição foi feita em relação ao grau relativo da planta.

\section{MRAC CONVENCIONAL}

Considere um polinômio $L(s)$ de grau $N=n^{*}-1$ escolhido de forma que $M(s) L(s)$ seja ERP (Estritamente Real Positiva).

Seja o sinal auxiliar

$$
y_{a}=M L \theta_{2 n+1}\left(L^{-1} u-\theta^{T} L^{-1} \omega\right)
$$

onde $\theta_{2 n+1}$ e $\theta$ são estimativas para $1 / \theta_{2 n}^{*}$ e $\theta^{*}$ (parâmetros de "Matching"), respectivamente. O erro aumentado é definido como

$$
e_{a}=\left(y-y_{m}\right)-y_{a}=e_{0}-y_{a} .
$$

Para garantir a estabilidade global do sistema adaptativo, Narendra, Lin e Valavani (1980) propuseram a seguinte modificação em $\mathrm{y}_{a}$

$$
\begin{aligned}
y_{a}=M L\left[\theta_{2 n+1}\left(L^{-1} \theta^{T}-\theta^{T} L^{-1}\right) \omega+\right. \\
\left.\alpha e_{a}\left(L^{-1} \omega\right)^{T}\left(L^{-1} \omega\right)\right], \quad \alpha>0 .
\end{aligned}
$$

Para atualizar $\theta(t)$ e $\theta_{2 n+1}(t)$ são usadas as seguintes leis integrais de adaptação

$$
\begin{gathered}
\dot{\theta}=-e_{a}\left(L^{-1} \omega\right) \\
\dot{\theta}_{2 n+1}=e_{a}\left(L^{-1} \theta^{T}-\theta^{T} L^{-1}\right) \omega .
\end{gathered}
$$

Assim, o MRAC convencional pode ser resumido na Tabela 1 .

Ioannou e Kokotovic (1984) contribuiram significativamente para o controle adaptativo de sistemas com grau relativo unitário, introduzindo o fator $\sigma$ na lei de adaptação

e, na forma entrada saída,

$$
\dot{\theta}=-\sigma \theta-e_{0} \omega
$$


Tabela 1: MRAC convencional.

\begin{tabular}{c}
$u=\theta^{T} \omega$ \\
\hline$e_{a}=\left(y-y_{m}\right)-y_{a}=e_{0}-y_{a}$ \\
$y_{a}=M L\left\lfloor\theta_{2 n+1}\left(L^{-1} \theta^{T}-\theta^{T} L^{-1}\right) \omega+\right.$ \\
$\left.\alpha e_{a}\left(L^{-1} \omega\right)^{T}\left(L^{-1} \omega\right)\right\rfloor, \alpha>0$ \\
$\dot{\theta}=-e_{a}\left(L^{-1} \omega\right)$ \\
$\dot{\theta}_{2 n+1}=e_{a}\left(L^{-1} \theta^{T}-\theta^{T} L^{-1}\right) \omega$ \\
\hline
\end{tabular}

a qual garante no mínimo estabilidade local na presença de dinâmica não modelada e/ou distúrbios externos. O primeiro termo do lado direito da igualdade pode ser interpretado como um fator de esquecimento e o segundo termo, um fator de aprendizagem. A introdução do fator $\sigma$, entretanto, pode levar ao aparecimento de "bursting" como verificado por Hsu e Costa (1987). Narendra e Annaswammi (1987) propuseram a utilização de um fator de esquecimento variável, substituindo o parâmetro sigma pelo módulo do erro de saída eliminando o efeito de "bursting".

A estabilidade global e a eliminação de "bursting" do algoritmo com modificação sigma foram obtidas depois por Ioannou e Tsakalis (1986), retendo a modificação sigma e introduzindo normalização no termo $e_{0} \omega$.

A introdução da normalização pode levar a transitórios de adaptação demasiadamente lentos e mesmo com a excitação rica em freqüências, a qualidade do transitório de adaptação (quando $\theta(t)$ está distante de $\theta^{*}$ ) não é uniforme e a convergência dos parâmetros adaptativos é muito lenta. Apesar do comportamento transitório não ser totalmente aceitável, em algumas situações, o sinal de controle é suave, tornando-o adequado para a condição de regime permanente.

\section{VS-MRAC}

O VS-MRAC foi desenvolvido por Hsu e Costa (1989) no intuito de buscar um controlador robusto em relação às incertezas da planta e com um desempenho transitório significativamente melhor que os obtidos com os algoritmos baseados em identificação de parâmetros. Para o caso de $n^{*}=1$, a solução foi substituir as leis integrais de adaptação por leis de adaptação a estrutura variável, tornando o erro de saída $e_{0}(t) \equiv 0$ uma superfície deslizante no espaço de estado do erro do sistema. A lei de adaptação é dada por

$$
\theta=-\bar{\Theta} \operatorname{sgn}\left(e_{0} \omega\right), \quad \bar{\Theta}=\operatorname{diag}\left(\bar{\theta}_{1} \ldots \bar{\theta}_{2 n}\right)
$$

onde $\bar{\theta}_{j}>\left|\theta_{j}^{*}\right|, j=1, \ldots, 2 n$.
Para o caso de $n^{*} \geq 2$ (Hsu (1990), Hsu Araújo e Costa(1994)), foi necessária a introdução de uma cadeia de erros auxiliares para o rastreamento do modelo. $\mathrm{O}$ algoritmo apresentado em Hsu (1990) foi modificado por Araújo e Hsu (1990), diminuindo o número de relés e possibilitando a obtenção de um melhor desempenho transitório.

Aqui será apresentada a versão compacta do VS-MRAC proposta por Araújo e Hsu (1990), denominada de VS-MRAC compacto.

Sejam os seguintes sinais filtrados

$$
\begin{gathered}
\xi_{i}=\left(L_{i+1} \ldots L_{N}\right)^{-1} \omega, \quad i=0,1, \ldots, N-1 \\
\xi_{N}=\omega \\
\chi_{i}=\left(L_{i+1} \ldots L_{N}\right)^{-1} u, i=0,1, \ldots, N-1 \\
\chi_{N}=u
\end{gathered}
$$

onde

$$
\begin{gathered}
L(s)=L_{1}(s) \ldots L_{N}(s) \quad, N=n^{*}-1 \\
L_{i}(s)=s+\delta_{i}, \quad \delta_{i}>0, \quad i=1, \ldots, N, \quad \delta_{i} \in \Re
\end{gathered}
$$

Define-se os limitantes superiores

$$
\begin{aligned}
& \underset{i j}{\bar{\theta}}>\rho\left|\theta_{j}^{*}-\theta_{j, n o m}\right|, \quad i=0,1, \ldots, N-1, j=1, \ldots, 2 n \\
& \underset{N j}{\bar{\theta}}>\left|\theta_{j}^{*}-\theta_{j, n o m}\right|, \quad j=1, \ldots, 2 n \\
& \bar{k}>\frac{\left|k^{*}-k_{\text {nom }}\right|}{k_{\text {nom }}}
\end{aligned}
$$

onde $\theta_{\text {nom }}$ é um vetor de parâmetros nominais obtido com algum modelo nominal da planta (idealmente $\theta_{j, n o m}=\theta_{j}^{*}$ ), $\rho=\frac{k^{*}}{k_{n o m}}$ (idealmente $\rho=1$ ), e $k_{n o m}$ é um valor nominal para $k^{*}=1 / \theta_{2 n}^{*}$ (assume-se $k_{n o m} \neq 0$ ). Além disso tem-se $u_{\text {nom }}=\theta_{n o m}^{T} \omega$.

O algoritmo de controle encontra-se resumido na Tabela 2.

As leis de adaptação a estrutura variável são escolhidas de forma que os erros auxiliares $e_{i}^{\prime}(i=0,1, \ldots, N)$ atinjam modos deslizantes em um tempo finito. Os controles equivalentes 
Tabela 2: VS-MRAC compacto.

\begin{tabular}{c}
\hline$u=-u_{N}+u_{n o m}$ \\
\hline$y_{a}=k_{n o m} M L\left\lfloor u_{0}-L^{-1}\left(u_{N}\right)\right\rfloor$ \\
$e_{a}=e_{0}-y_{a}$ \\
$e_{0}^{\prime}=e_{a}$ \\
$e_{i}^{\prime}=\left(u_{i-1}\right)_{e q}^{*}-L_{i}^{-1}\left(u_{i}\right), i=1, \ldots, N$ \\
\hline$f_{i}=\bar{k}\left|\chi_{i}-\theta_{n o m}^{T} \xi_{i}\right|+\bar{\theta}_{i}^{T}\left|\xi_{i}\right|, \quad i=0,1, \ldots, N-1$ \\
$f_{N}=\bar{\theta}_{N}\left|\chi_{N}\right|, \bar{\theta}_{i}^{T}=\left[\bar{\theta}_{i 1} \ldots \bar{\theta}_{i, 2 n}\right], \quad i=0, \ldots, N$ \\
$u_{i}=f_{i} \operatorname{sgn}\left(e_{i}^{\prime}\right), \quad i=0,1, \ldots, N$ \\
\hline
\end{tabular}

$\left(\left(u_{i}\right)_{e q}^{*}\right)$ são obtidos assintoticamente de $\left(u_{i}\right)$ por meio de um filtro passa-baixas com freqüência de corte suficientemente elevada.

\section{DMARC}

\section{1 caso $n^{*}=1$}

No DMARC é feita uma ligação entre o MRAC e o VSMRAC através da variação em tempo real de um parâmetro na lei de adaptação. Considere a seguinte lei de adaptação

$$
\mu \dot{\theta}=-\sigma \theta-\sigma \Gamma e_{o} \omega
$$

onde

$$
\Gamma=\operatorname{diag}\left[\frac{\bar{\theta}_{j}}{\left|e_{o} \omega_{j}\right|}\right], \quad \bar{\theta}_{j}>\left|\theta_{j}^{*}\right| \quad, j=1, \ldots, 2 n .
$$

Quando $\mu \rightarrow 0$, nota-se que a equação (17) se resume a (16), ou seja, ao algoritmo VS-MRAC. A equação (17) pode ser reescrita como

$$
\dot{\theta}=-\frac{\sigma}{\mu} \theta-\frac{1}{\mu} \sigma \Gamma e_{o} \omega .
$$

Novamente, quando $\mu \rightarrow 0$, observa-se que o fator de esquecimento tende a infinito, implicando que o VS-MRAC não tem memória. $\mathrm{O}$ termo de aprendizagem também cresce ilimitadamente, de onde conclui-se que no VS-MRAC a adaptação é instantânea.

Quando $\mu=1$, a equação (17) se resume a

$$
\dot{\theta}=-\sigma \theta-\sigma \Gamma e_{o} \omega
$$

que é a lei de adaptação do MRAC com fator $\sigma$ e uma normalização no termo de aprendizagem.

\section{2 caso $\mathrm{n}^{\star} \geq 2$}

De forma similar ao artigo de Hsu e Costa (1994), usa-se a estrutura do VS-MRAC, descrita na Tabela 2, que é aplicável para $\mathbf{n}^{*} \geq 2$. Utiliza-se o DMARC na última malha responsável pela geração do sinal de controle $\mathbf{u}_{\mathbf{N}}$ (ver Figura 1).

Tabela 3: DMARC.

\begin{tabular}{c}
$u=-u_{N}+u_{\text {nom }}$ \\
\hline$y_{a}=k_{n o m} M L\left\lfloor u_{0}-L^{-1}\left(u_{N}\right)\right\rfloor$ \\
$e_{a}=e_{0}-y_{a}$ \\
$e_{0}^{\prime}=e_{a}$ \\
$e_{i}^{\prime}=\left(u_{i-1}\right)_{e q}^{*}-L_{i}^{-1}\left(u_{i}\right), \quad i=1, \ldots, N$ \\
\hline$f_{i}=\bar{k}\left|\chi_{i}-\theta_{n o m}^{T} \xi_{i}\right|+\bar{\theta}_{i}^{T}\left|\xi_{i}\right|, i=0,1, \ldots, N-1$ \\
$u_{i}=f_{i} \operatorname{sgn}\left(e_{i}^{\prime}\right), \quad i=0,1, \ldots, N-1$ \\
$u_{N}=-\theta_{N}^{T} \omega$ \\
$\mu \dot{\theta}_{N}=-\sigma \theta_{N}-\sigma \Gamma_{N} e_{N}^{\prime} \omega$ \\
\hline
\end{tabular}

O algoritmo de controle é apresentado na Tabela 3. Na Figura 1, o bloco pontilhado que representa o relé regido pela função de modulação $\mathbf{f}_{\mathbf{N}}$ é substituido por leis adaptativas ou chaveadas (geradas pelo DMARC) de forma que $\mathbf{u}_{\mathbf{N}}$ é determinado pelas expressões

$$
\begin{gathered}
u_{N}=-\theta_{N}^{T} \omega \\
\mu \dot{\theta}_{N}=-\sigma \theta_{N}-\sigma \Gamma_{N} e_{N}^{\prime} \omega
\end{gathered}
$$

onde

$$
\Gamma_{N}=\operatorname{diag}\left[\frac{\bar{\theta}_{N, j}}{\left|e_{N}^{\prime} \omega_{j}\right|}\right], j=1, \ldots, 2 n .
$$

Analisando as duas últimas expressões da Tabela 3, vê-se que quando $\mu \rightarrow 0$

$$
\theta_{N}=-\bar{\Theta}_{N} \operatorname{sgn}\left(e_{N}^{\prime} \omega\right), \quad \bar{\Theta}_{N}=\operatorname{diag}\left(\bar{\theta}_{N, 1} \ldots \bar{\theta}_{N, 2 n}\right)
$$

e, como $\xi_{N}=\omega$ tem-se para $u_{N}$

$$
u_{N}=(\operatorname{sgn}(\omega))^{T} \bar{\Theta}_{N} \omega \operatorname{sgn}\left(e_{N}^{\prime}\right)
$$




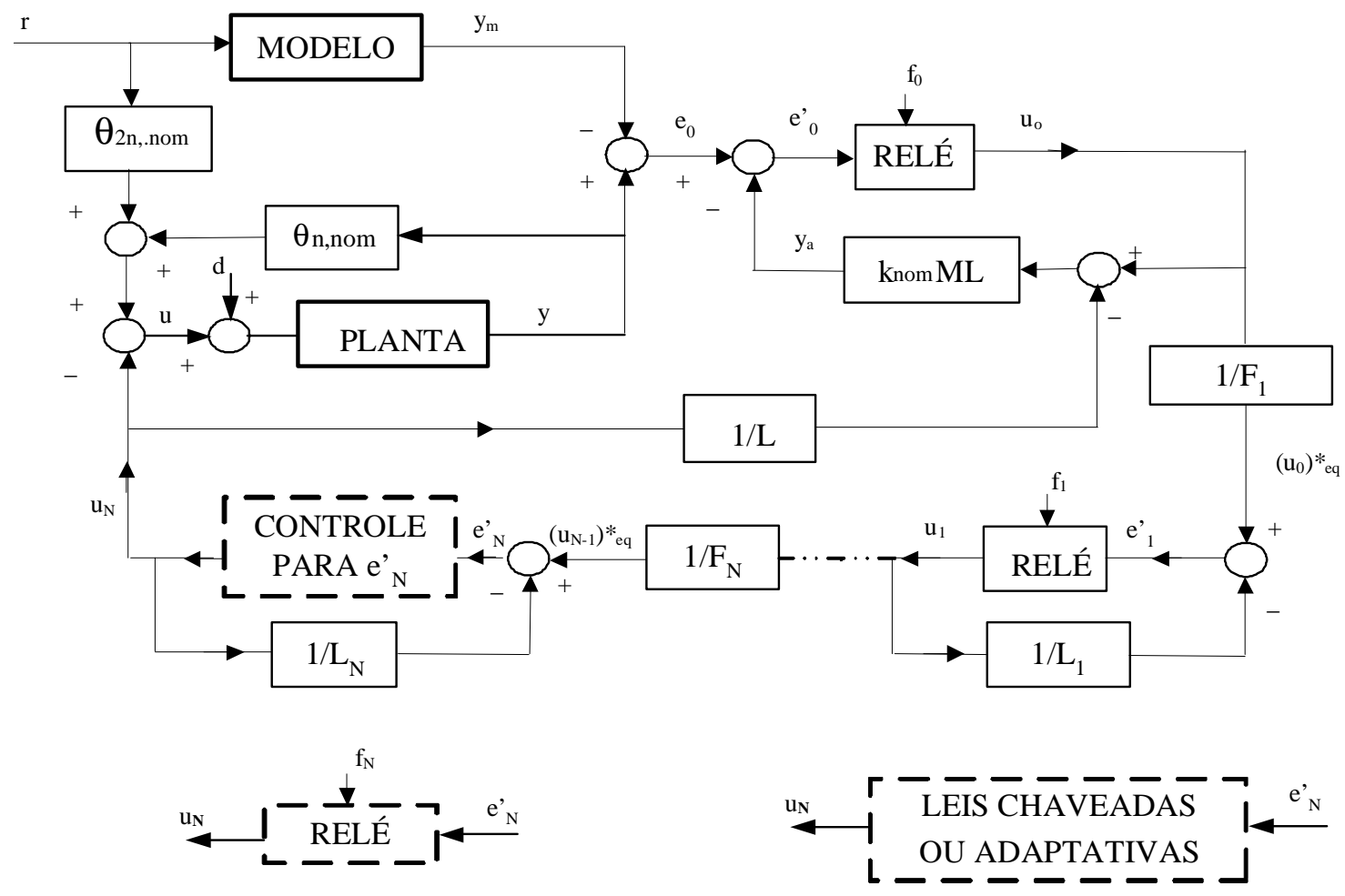

Controle VS-MRAC para e' ${ }_{N}$

Controle DMARC para e' ${ }_{\mathrm{N}}$

Figura 1: Diagrama de blocos do VS-MRAC e do DMARC.

$$
\begin{gathered}
u_{N}=\bar{\theta}_{N}^{T}|\omega| \operatorname{sgn}\left(e_{N}^{\prime}\right) \\
u_{N}=f_{N} \operatorname{sgn}\left(e_{N}^{\prime}\right)
\end{gathered}
$$

que é o resultado das duas últimas expressões do VS-MRAC da Tabela 2.

\section{OBTENÇÃO DO PARÂMETRO $\mu$}

Para o ajuste do DMARC é importante que as transições entre os controladores MRAC e VS-MRAC se dêem de uma forma suave e contínua. No artigo de Cunha e outros (2005) foi utilizado o DMARC no controle de velocidade de um motor de indução trifásico. O cálculo do parâmetro $\mu$, da lei de adaptação do DMARC, foi feito com a utilização de lógica nebulosa, pelo modelo de inferência Mandani, sendo as variáveis lingüísticas de entrada, o erro de saída e a sua derivada. No artigo Mota e Araújo (2002), o parâmetro $\mu$ foi usado no sinal de controle, ponderando a ação de cada controlador (MRAC e VS-MRAC). Na determinação do parâmetro $\mu$ foi usada a lógica nebulosa com modelo de inferên- cia Takagi-Sugeno (Takagi e Sugeno (1985)), tendo apenas o erro de saída como variável lingüística de entrada.

Nesse trabalho, para plantas com $n^{*}=1$, a determinação do parâmetro é feita, usando a expressão

$$
\mu=e^{\frac{-e_{0}^{2}}{\ell}}
$$

onde $e_{0}$ é o erro de saída e $\ell$ é um parâmetro a ser ajustado. Essa expressão é similar às usadas como função de pertinência por Mota e Araújo (2002). Observa-se, da equação (23), que quando $e_{0} \rightarrow 0, \mu \rightarrow 1$ aproximando-se do MRAC. Quando $e_{0}$ se torna razoavelmente elevado $\mu$ assume um valor suficientemente pequeno tendendo ao VSMRAC. O parâmetro $\ell$ tem a importante função de determinar a forma como se dá a transição entre o MRAC e o VS-MRAC. Quanto menor o valor de $\ell$ maior a ação do VSMRAC em função de $e_{0}$, como visto na Figura 2.

A forma como é feita a transição do VS-MRAC para o MRAC, ou seja, o compromisso entre rapidez de resposta (VS-MRAC) e sinal suave em regime permanente (MRAC) fica a critério da escolha do parâmetro $\ell$. 


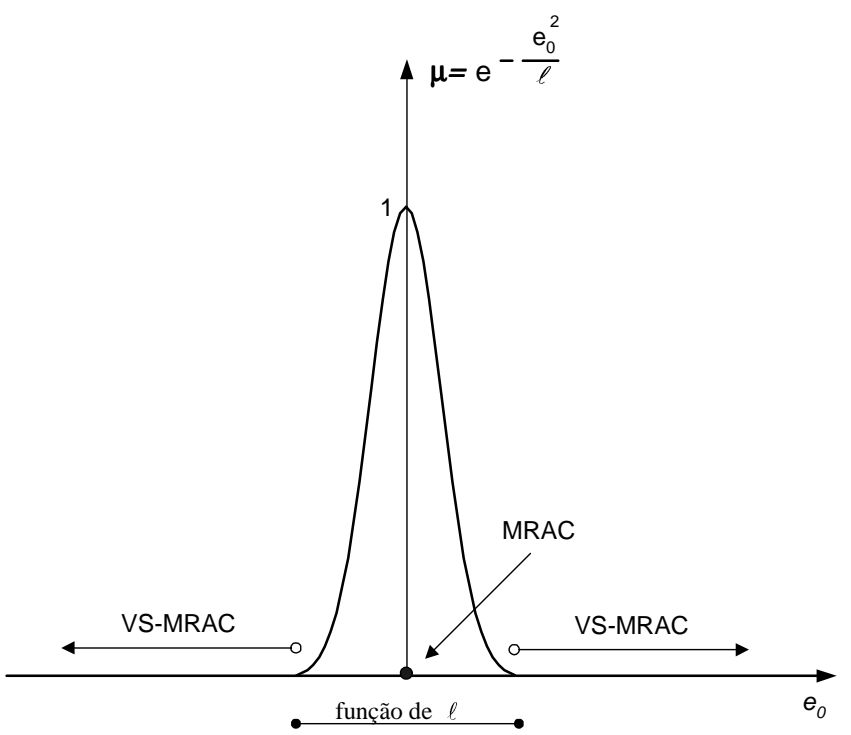

Figura 2: Evolução de $\mu$ em função do erro de saída.

Analisando a derivada da equação (23) tem-se

$$
\dot{\mu}=-2 \frac{\mu}{\ell} e_{0} \dot{e}_{0} .
$$

Sendo $e_{0}=0$ a superfície de deslizamento e $e_{0} \dot{e}_{0}<0$, a condição de escorregamento para o VS-MRAC, vê-se que o parâmetro $\mu$ só pode crescer (tendendo ao MRAC) quando a condição de escorregamento for satisfeita. Se a condição de escorregamento não for satisfeita o $\mu$ decresce (tendendo ao VS-MRAC) tão mais rápido quanto menor for o parâmetro $\ell$. Com um valor de $\ell$ suficientemente pequeno, o algoritmo de controle funciona como VS-MRAC até um valor bem pequeno de $e_{0}$, permitindo uma resposta transitória muito rápida.

Tem-se, então, a análise apresentada na Tabela 4.

Tabela 4: Comportamento de $\mu$ quanto à condição de escorregamento.

\begin{tabular}{|c|c|}
\hline $\begin{array}{c}\text { Se } e_{0} \dot{e}_{0}<0 \\
\text { condição de } \\
\text { escorregamento satisfeita }\end{array}$ & $\begin{array}{c}\text { então } \dot{\mu}>0 \\
\mu \text { aumenta (aproxima-se } \\
\text { do MRAC) }\end{array}$ \\
\hline $\begin{array}{c}\text { Se } e_{0} \dot{e}_{0}>0 \\
\text { condição de } \\
\text { escorregamento não } \\
\text { satisfeita }\end{array}$ & $\begin{array}{c}\mu \text { diminuĩo } \dot{\mu}<0 \\
\text { do VS-MRAC) }\end{array}$ \\
\hline $\begin{array}{c}\text { Se } e_{0} \dot{e}_{0}=0 \\
\text { superfície de }\end{array}$ & $\begin{array}{c}\text { então } \dot{\mu}=0 \\
\text { escorregamento alcançada }\end{array}$ \\
$\begin{array}{c}\mu \text { constante, controlador } \\
\text { fixo }\end{array}$ \\
\hline
\end{tabular}

\section{ANÁLISE DE ESTABILIDADE PARA PLANTAS DE PRIMEIRA ORDEM}

Considere um exemplo simples onde a planta e o modelo de referência são dados, respectivamente, por

$$
\begin{gathered}
\dot{y}=-a y+u ; \quad y, u \in \Re ; \quad a \in \Re \\
\dot{y}_{m}=-a_{m} y_{m}+r ; \quad y_{m}, r \in \Re ; \quad a_{m} \in \Re, \quad a_{m}>0
\end{gathered}
$$

onde $a$ é o único parâmetro desconhecido.

A lei de adaptação do DMARC (17) é

$$
\mu \dot{\theta}=-\sigma \theta-\sigma \bar{\theta} \operatorname{sgn}\left(e_{0} y\right)
$$

com $\mu$ dado por (23). A lei de controle é

$$
u=\theta y+r .
$$

No modo regulação $(r=0), y_{m}=0, e_{0}=y$ e uma realização para o erro de saída é escrita como segue

$$
\begin{gathered}
\dot{e}_{0}=(\theta-a) e_{0} \\
\dot{\theta}=-\sigma e^{\frac{e_{0}^{2}}{\ell}}(\theta+\bar{\theta}) \quad, \quad \bar{\theta}>|a|+\varepsilon, \quad \varepsilon>\frac{1}{4} \text { ou } \\
\dot{\hat{\theta}}=-\sigma e^{\frac{e_{0}^{2}}{\ell} \hat{\theta}}, \quad \hat{\theta}=\theta+\bar{\theta} .
\end{gathered}
$$

Nota-se que o sistema possui um ponto de equilíbrio em $\left(e_{0}=0, \hat{\theta}=0\right)$, cuja análise linearizada, em torno desse ponto de equilíbrio, caracteriza-o como um nó estável.

Definindo a função de Lyapunov

$$
V=\frac{1}{2} e_{0}^{2}+\frac{1}{2 \sigma} \hat{\theta}^{2}
$$

tem-se para sua derivada

$$
\dot{V}=-(\bar{\theta}+a) e_{0}^{2}+\hat{\theta} e_{0}^{2}-\hat{\theta}^{2} e^{\frac{e_{0}^{2}}{\ell}} .
$$

Analisando (31), nota-se que para $\hat{\theta} \leq 0$ tem-se $\dot{V}<0$. Considerando $0<\ell \leq 1$, tem-se que $e^{\frac{e_{0}^{2}}{\ell}}>e_{0}^{2}$. Assim, para 


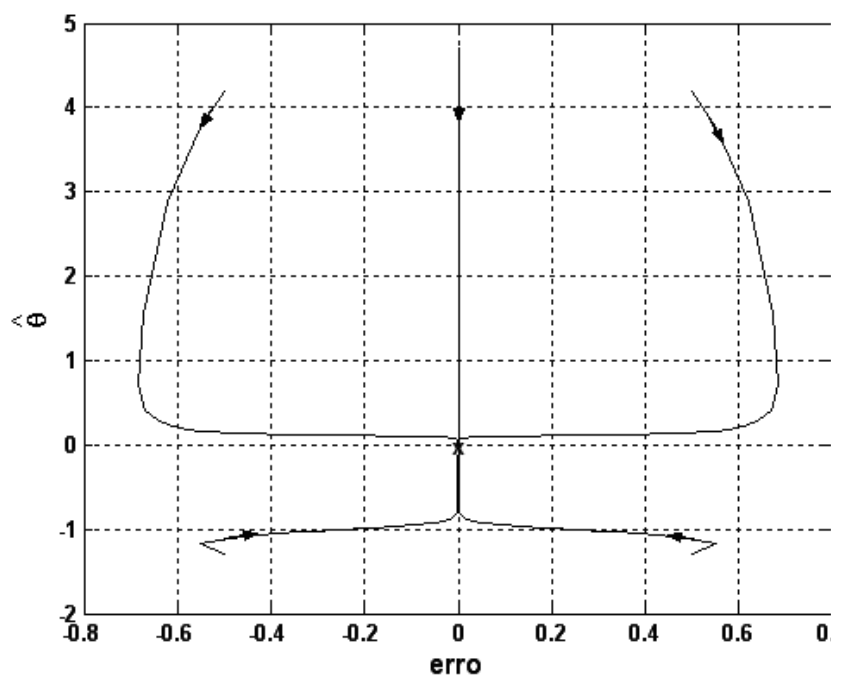

Figura 3: Plano de fase para uma planta de primeira ordem, $r=0$.

$\hat{\theta} \geq 1$ implica que $\hat{\theta}^{2} \geq \hat{\theta}$ e, conseqüentemente, $\dot{V}<0$. Ainda, a partir de (31) tem-se

$$
\dot{V} \leq-(\bar{\theta}+a) e_{0}^{2}+\left(\hat{\theta}-\hat{\theta}^{2}\right) e_{0}^{2}
$$

No intervalo $0<\hat{\theta}<1$, o segundo termo do lado direito de (32) é positivo. Porém, a função $\left(\hat{\theta}-\hat{\theta}^{2}\right)$ possui um máximo em $\hat{\theta}=\frac{1}{2}$. Desta forma, reescreve-se (32) como

$$
\dot{V} \leq\left(-\bar{\theta}-a+\frac{1}{4}\right) e_{0}^{2}
$$

Com esse resultado, de (29) e (31), vê-se que $\dot{V}=0$ apenas no ponto $\left(e_{0}=0, \hat{\theta}=0\right)$ e $\dot{V}<0$ para todo espaço em torno dele. Como $V>0$ é radialmente ilimitada, conclui-se que $\left(e_{0}=0, \hat{\theta}=0\right)$ é globalmente assintoticamente estável. Devido à estabilidade assintótica global da origem no espaço de estado, conclui-se que para esse controlador não há possibilidade do surgimento do fenômeno de "bursting".

Na Figura 3 é apresentado o plano de fase para $r=0$, com cinco condições iniciais distintas. Observa-se a convergência para $\left(e_{0}=0, \hat{\theta}=0\right)$ independente da condição inicial. Os dados utilizados na simulação foram: $a=-2, a_{m}=1$, $\bar{\theta}=3,2, \ell=0,1$ e $\sigma=0,1$. Os resultados das simulações para $r=0$ encontram-se na Figura 3.

Com os mesmos dados anteriores foram feitas simulações para $r=1$ e os resultados encontram-se na Figura 4. Nesse caso, como a referência é persistentemente excitante,

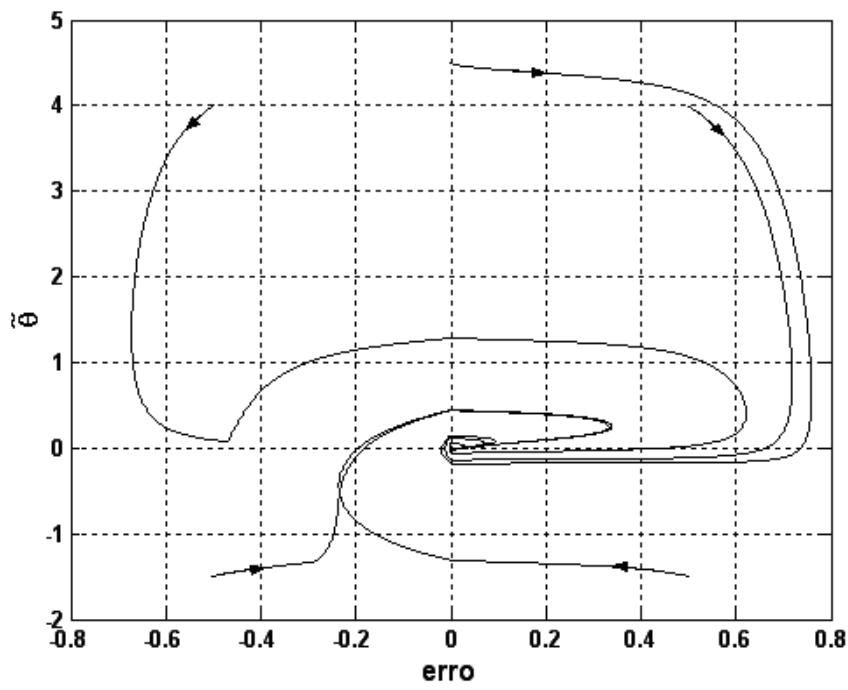

Figura 4: Plano de fase para uma planta de primeira ordem, $r=1$.

observam-se as convergências de $\theta$ para $\theta^{*}(\tilde{\theta}=0)$ e do erro de saída para zero.

A análise de estabilidade, para plantas $\operatorname{com} n^{*}>1$, pode ser feita a partir da análise de plantas com $n^{*}=1$, seguindo Hsu (1990) e Hsu e Costa (1994). Uma vez que $e_{N}^{\prime}$ é governado por uma função de transferência estritamente real positiva (ver Figura 1 e Tabela 3) é sugerido o uso da lei de adaptação do DMARC para $n^{*}=1$, no bloco pontilhado da Figura 1, onde é gerado $u_{N}$. Desde que seja garantido que $\theta_{N}$ é uniformemente limitado, todos os sinais do sistema serão uniformemente limitados. Assim, independente da forma como é feita a modificação em $\theta_{N}$, a convergência exponencial dos erros auxiliares $e_{i}^{\prime}(i=0,1, \ldots, N-1)$ para zero permanece válida (Hsu (1990) e Hsu e Costa (1994)).

\section{RESULTADOS DE SIMULAÇÕES}

Foi escolhido o controle de posição de um motor CC cuja função de transferência é um sistema de ordem e grau relativo, ambos, iguais a 3 (Feller e Benz (1987)). A função de transferência da planta é

$$
W(s)=\frac{2.10^{3} \hat{k}_{p}}{s\left(s^{2}+20 s+2000\right)}
$$

onde $\hat{k}_{p}$ varia entre 2,5 e 10 , tornando o ganho de alta frequiência conhecido com incertezas.

O modelo de referência é especificado por 


$$
M(s)=\frac{10^{4}}{(s+55,55)^{3}}
$$

e os sinais $v_{1}$ e $v_{2}$ com a mesma dinâmica do modelo, ou seja

$$
\Lambda=\left[\begin{array}{cc}
0 & 1 \\
-3090 & -111
\end{array}\right] \quad ; \quad g=\left[\begin{array}{c}
0 \\
3090
\end{array}\right]
$$

Foi escolhido o polinômio L(s) como

$$
L(s)=L_{1}(s) L_{2}(s)=(s+55,55)^{2}
$$

tornando, assim, mais simples o operador ML.

Para gerar os controles equivalentes foram usados os seguintes filtros de segunda ordem

$$
\begin{gathered}
F_{i}(s)=\left(\tau_{i} s\right)^{2}+2 \xi \tau_{i} s+1 \\
\tau_{i}=(400 \pi)^{-1}, \quad \xi=0,7 \quad, \quad i=1,2 .
\end{gathered}
$$

Os parâmetros do controlador para o VS-MRAC compacto determinados a partir de $\theta^{*}$ e da incerteza em $\hat{k}_{p}$ são

$$
\begin{gathered}
\theta_{\text {nom }}^{T}=(0 ; 0 ; 0 ; 0 ; 0 ; 1) ; k_{n o m}=1 \\
\bar{\theta}_{i}^{T}=(13,5 ; 0,1 ; 75 ; 60 ; 2,75 ; 1,1) ; i=0,1 \\
\bar{\theta}_{2}^{T}=(6,75 ; 0,05 ; 150 ; 120 ; 5,5 ; 1,1) ; \bar{k}=1,1 .
\end{gathered}
$$

Inicialmente foram feitas simulações com o MRAC Convencional e o VS-MRAC compacto com $\hat{k}_{p}=5$ e $r(t)=0,1$ mantidos constantes. As condições iniciais foram tais que $y(0)=2 \cdot 10^{-3}$. Os resultados dessas simulações com o MRAC encontram-se na Figura 5 e com o VS-MRAC na Figura 6.

Verifica-se no MRAC um transitório demasiadamente longo e oscilatório (comparado com o VS-MRAC), porém com sinal de controle suave. No VS-MRAC o transitório é rápido, pouco oscilatório, mas com o sinal de controle de alta freqüência.

Nas simulações subseqüentes, foram feitas as modificações mostradas na Tabela 5 .

Para sintonizar o DMARC utilizou-se a expressão para o parâmetro $\mu$

$$
\mu=e^{-e_{N}^{\prime 2} / \ell}
$$
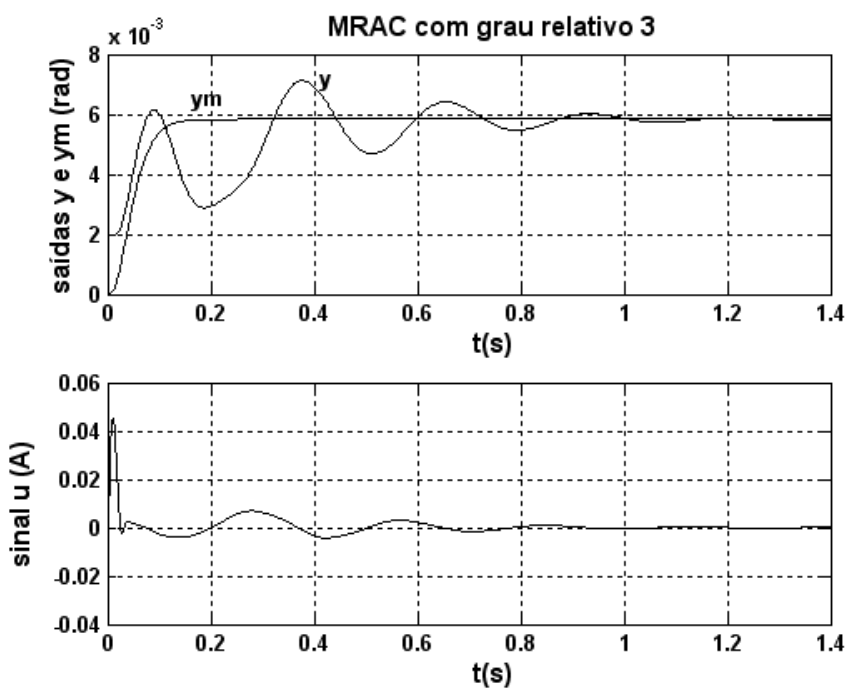

Figura 5: Desempenho do MRAC.

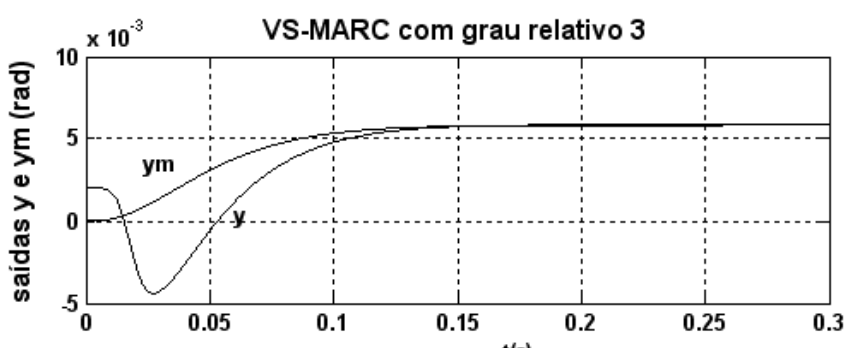

$t(s)$

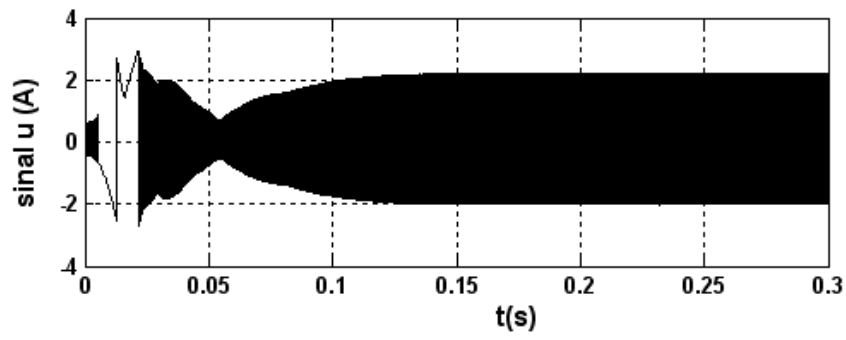

Figura 6: Desempenho do VS-MRAC.

Tabela 5: Modificações feitas nas simulações

\begin{tabular}{|c|c|c|}
\hline \multicolumn{3}{|c|}{ para $0 \mathrm{~s} \leq \mathrm{t}<0,2 \mathrm{~s}$} \\
\hline$r(t)=0,1$ & $d(t)=0,03$ & $\hat{k}_{p}=5$ \\
\hline \multicolumn{3}{|c|}{ para $0,2 \mathrm{~s} \leq \mathrm{t}<0,4 \mathrm{~s}$} \\
\hline$r(t)=0$ & $d(t)=0$ & $\hat{k}_{p}=10$ \\
\hline \multicolumn{3}{|c|}{ para $0,4 \mathrm{~s} \leq \mathrm{t} \leq 0,6 \mathrm{~s}$} \\
\hline$r(t)=0,1$ & $d(t)=-0,03$ & $\hat{k}_{p}=2,5$ \\
\hline
\end{tabular}



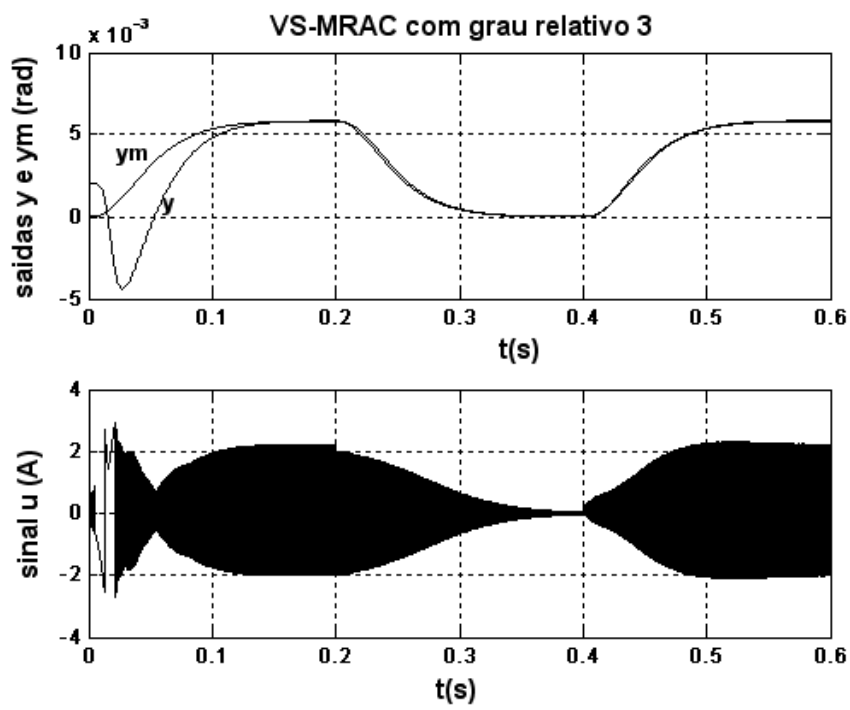

Figura 7: Desempenho do VS-MRAC.
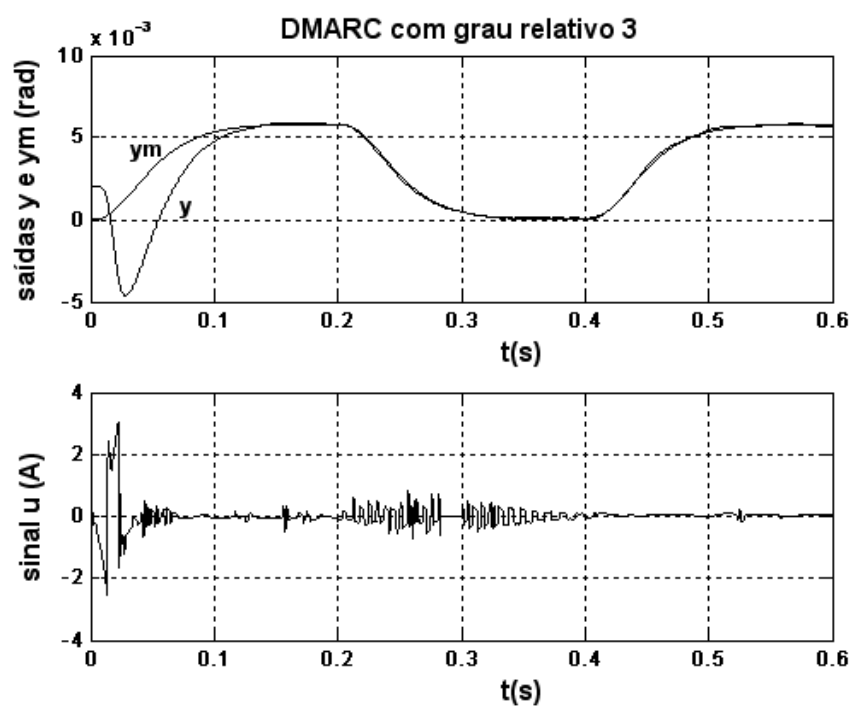

Figura 8: Desempenho do DMARC.

onde $\ell$ é um parâmetro a ser ajustado.

Nessas novas condições foram escolhidos os parâmetros $\sigma=$ 0,05 e $\ell=5 \cdot 10^{-9}$ para o DMARC.

Os resultados das simulações com o VS-MRAC e com o DMARC são apresentados nas Figuras 7 e 8, respectivamente. Nota-se que tanto o VS-MRAC como o DMARC apresentam uma pequena oscilação, atingindo o regime permanente em um tempo consideravelmente pequeno, com robustez em relação à variação paramétrica e à perturbação externa. Entretanto, o sinal de controle do DMARC apresenta uma boa suavização, em relação ao sinal do VS-MRAC, e pequena magnitude em regime permanente.
A análise de $e_{N}^{\prime}$ em (34) é semelhante à análise feita para $e_{0}$ em (23), para o caso $n^{*}=1$, uma vez que a malha na qual se encontra $e_{N}^{\prime}$ é representada por um sistema de primeira ordem.

\section{CONCLUSÕES}

Neste artigo é proposto um DMARC para o controle de uma planta com grau relativo arbitrário. Utilizou-se a estrutura do VS-MRAC compacto proposta por Araújo e Hsu (1990). Conforme pode ser verificado pelas simulações, o algoritmo em Modo Dual Adaptativo Robusto proporcionou um transitório rápido e praticamente sem oscilações e um desempenho em regime permanente com sinal de controle com boa suavização. Adicionalmente apresentou robustez a incertezas paramétricas e distúrbios. Uma análise de estabilidade é feita para o caso particular de uma planta de primeira ordem. A análise generalizada, para plantas com grau relativo arbitrário, poderá ser feita seguindo Hsu (1990) e Hsu e Costa (1994), considerando-se que na Figura 1 o DMARC é aplicado na última malha, a um sistema de primeira ordem.

\section{REFERÊNCIAS}

Andrievskii, B. R., Stotskii, A. A. e Fradkov, A. L. (1988). Velocity Gradient Algorithms in Control and Adaptation Problems, Automation and Remote Control, 49(12), Part 1, 1533-1564.

Araújo, A. D. e Hsu, L. (1990). Further Developments in Variable Structure Adaptive Control Based only on Input/Output Measurements, Proceedings of the $11^{\text {th }}$ IFAC World Congress, Tallinn, August, 4, 293-298.

Åström, K. J. e Wittenmark, B. (1995). Adaptive Control, Addison-Wesley Pub. Co.

Cunha, C. D., Araújo, A. D., Barbalho, D. S. e Mota, F. C. (2005). A Dual-Mode Adaptive Robust Controller Applied to the Speed Control of a Three-Phase Induction Motor, Asian Journal of Control, 7(2), 197-201.

Cunha, J.P.V.S., Hsu, L., Costa, R.R. e Lizarralde, F. (2003). Output-Feedback Model-Reference Sliding Mode Control of Uncertain Multivariable Systems, IEEE Trans. Automatic Control, AC-48 (12), 2245-2250.

Emelyanov, S. V. (1987). Binary Automatic Control Systems. MIR Publishers, Moscow (English Translation).

Feller, P. e Benz, U. (1987). Sliding Mode Position Control of a DC Motor, Proc. 10th IFAC World Congress, 3, 333-338.

Hsu, L. (1990). Variable Structure Model Reference Adaptive Control Using only Input and Output Measure- 
ments: The General Case, IEEE Trans. Automatic Control, AC- 35 (11), 1238-1243.

Hsu, L. e Costa, R.R. (1987). Bursting Phenomena in Continuous-Time Adaptive Systems with a $\sigma$ Modification, IEEE Trans. Automatic Control, AC32(1), 84-86.

Hsu, L. e Costa, R. R. (1989). Variable Structure Model Reference Adaptive Control Using only Input and Output Measurements: Part I, Int. J. Control, 49(2), 399-416.

Hsu, L., Araújo, A. D. e Costa, R. R. (1994). On the Design of Variable Structure Adaptive Control Using only Input/ Output Data, IEEE Trans. Automatic Control, AC39 (1), 4-21.

Hsu, L. e Costa, R.R. (1990). A Binary Control Approach to Design Globally Exponentially Stable Systems. Proceedings of the $7^{\text {th }}$ International Conference on Systems Engineering, Las Vegas, July.

Hsu, L. e Costa, R. R. (1994). B-MRAC Global Exponential Stability with a New Model Reference Adaptive Controller Based on Binary Control Theory, ControlTheory and Advanced Technology, 10 (4), Part 1, 649668.

Hsu, L., Lizarralde, F. e Araujo, A. D. (1997). New Results on Output-Feedback Variable Structure ModelReference Adaptive Control: Design and Stability Analysis, IEEE Trans. Automatic Control, AC-42 (3), 386-393

Ioannou, P. A. e Sun, J. (1996). Robust Adaptive Control, Prentice-Hall, Inc., Englewood Cliffs.

Ioannou, P. A. e Kokotovic, P. V. (1984). Instability Analysis and Improvement of Robustness of Adaptive Control, Automatica, 20(5),583-594

Ioannou, P. A. e Tsakalis, K. S. (1986). A Robust Direct Adaptive Controller, IEEE Trans. Automatic Control, AC-31( 11), 1033-1043.

Mota, F. C. e Araújo, A. D. (2002). Proposta de um Controlador Dual Adaptativo Robusto Utilizando o Modelo de Takagi-Sugeno, Congresso Brasileiro de Automática, Natal-RN, Brasil, 173-177.

Narendra, K. S. e Annaswamy, A. M. (1987). New Adaptive Law for Robust Adaptive Control Without Persistent Excitation, IEEE Trans Automatic Control, AC-32, $134-145$.

Narendra, K. S. e Annaswamy, A. M. (1989). Stable Adaptive Systems, Prentice Hall, Inc., New Jersey.
Narendra, K. S., Lin, Y. H. e Valavani, L. S. (1980). Stable Adaptive Controller Design - Direct Control, IEEE Trans. Automatic Control, AC-25( 6), 570-583.

Sastry, S. S. (1984). Model - Reference Adaptive Control Stability, Parameter Convergence and Robustness, IMA J. Mathematical Control and Information, 1, 27-66.

Sastry, S.S. e Bodson, M. (1989). Adaptive Control: Stability Convergence and Robustness, Englewood Cliffs, NJ, Prentice-Hall.

Takagi, T. e Sugeno, M. (1985). Fuzzy Identification of Systems and its Applications to Modeling and Control, IEEE Trans. Syst. Man and Cybern., SMC-15(1), 116132. 